\section{Association of Ischemic Brainstem Stroke Following Tick Bite and Crimean-Congo Hemorrhagic Fever: A Rare Presentation}

\section{Ersin Kasım Ulusoy}

Department of Neurology, Kayseri Training and Education Hospital, Kayseri, Turkey

*Corresponding author: Ersin Kasım Ulusoy, MD, Department of Neurology, Kayseri Training and Education Hospital, Kayseri, Turkey, Tel: Tel: 90 506 6689392 ; Email: ersinkasim_ulusoy@hotmail.com

Received date: January 04, 2018; Accepted date: January 12, 2018; Published date: January 15, 2018

Copyright: $@ 2018$ Ulusoy EK. This is an open-access article distributed under the terms of the Creative Commons Attribution License, which permits unrestricted use, distribution, and reproduction in any medium, provided the original author and source are credited.

\begin{abstract}
Purpose: Crimean-Congo hemorrhagic fever (CCHF) is a viral zoonosis that may be associated with high mortality rate. Several inflammatory mediators (interleukins, IFN- $\gamma$, TNF- $\alpha$ etc.) released during disease have been implied in the pathogenesis, which also cause ischemia.

Case report: A 60-years old man working in livestock production presented to emergency department with vomiting, fever and impaired balance and gait. In laboratory evaluation, it was found that there was elevation in hepatic enzymes, decreased platelet and leukocyte counts. The patient underwent magnetic resonance (MR) imaging due to gait disorder, revealing restricted diffusion at pons compatible with acute infarct. The patient was admitted with the diagnosis of CCHF and ischemic stroke secondary to infection. During follow-up, no hemorrhage was observed and the patient was discharged as he achieved clinical recovery.

Conclusion: In this case report, we discussed ischemic stroke developed secondary to infection in a patient with $\mathrm{CCHF}$ and its management in the light of literature.
\end{abstract}

Keywords: Crimean-Congo hemorrhagic fever; Tick; Ischemic stroke; Management

\section{Introduction}

Crimean-Congo hemorrhagic fever is a fatal tick-borne viral infection seen in Africa, Eastern Europe and Middle East regions. It is caused by Nairovirus belonging to Bunyaviridae family. It is transmitted to human via bite by infected ticks (Hyalomma species) or contact to blood or body fluids of patient with disease [1]. The individuals at risk for CCHF include agricultural workers, veterinaries, and healthcare personnel and military personnel working at endemic areas [2]. The CCHF is an acute, potentially fatal disorder involving many organs, which is characterized by diffuse ecchymosis, visceral bleeding and impaired hepatic functions. Its clinical presentations range from mild disease including symptoms such as fever, headache, sore throat, nausea or vomiting to severe disease progressing with serious bleeding, shock, altered mental status and even death. Early diagnosis and treatment is essential due to high mortality rate [3].

Systemic infections comprise risk factor for development of ischemic stroke. In CCHF, bleedings are major causes for mortality. The pathogenesis underlying CCHF hasn't been fully elucidated yet. However, several inflammatory mediators (interleukins, IFN- $\gamma$, TNF- $\alpha$ etc.) released during disease have been implied in the pathogenesis, which also cause ischemia $[4,5]$.

In this case report, we intended to discuss ischemic stroke developed secondary to infection in a patient with CCHF progressing with fatal bleeding and its management in the light of literature.

\section{Case Report}

A 60-years old man living in rural areas of Sivas province and working in livestock production presented to emergency department with headache, nausea, vomiting, fever, fatigue, abdominal pain over 3 days and additional complaints of imbalance and inability to stand which occurred after initial complaints. In Emergency Department (ED), vital signs were recorded as follows: blood pressure, 140/90 $\mathrm{mmHg}$; heart rate, $118 \mathrm{bpm}$; respiration rate, 15/min; and body temperature, $38.8^{\circ} \mathrm{C}$. In the patients past medical history he had diyabet and we learn that he wasn't take any medications. In physical examination, there was no pathological finding other than tachycardia. In neurological examination, the patient was conscious and cooperative with normal orientation. Pupillary light reflexes were bilaterally positive and pupils were isochoric with normal ocular movements. Uvula was localized at midline whilepalatal arcs showed normal movement and gag reflex was also normal. There was no motor weakness in upper and lower extremities. Cerebellar tests were normal in both sites at upper body while the patient was unable to walk due to truncal ataxia.

It was found that there was elevation in hepatic enzymes with decreased platelet and leukocyte counts in the laboratory tests performed at presentation to ED (Table 1). The patient was consulted with hematology and infectious disease departments due to presence of bicytopenia and fever. In addition, the patient underwent abdominal CT scan to exclude malignancy. There was no evidence of malignancy in the abdominal CT scan and peripheral blood smear. A cranial MR imaging was performed due to ataxic gait, which revealed acute restricted diffusion anterior to pons at left half localized at level of middle cerebellar peduncle (Figure 1). The patient assessed by infectious disease department was considered as CCHF and admitted 
Citation: Ulusoy EK (2018) Association of Ischemic Brainstem Stroke Following Tick Bite and Crimean-Congo Hemorrhagic Fever: A Rare Presentation. J Clin Res Bioeth 9: 319. doi:10.4172/2155-9627.1000319

Page 2 of 3

to hospital by taking precautions of isolation. When the patient was questioned for CCHF, it was found out that he works in livestock production but he had no history of tick exposure. No tick was detected in physical examination.
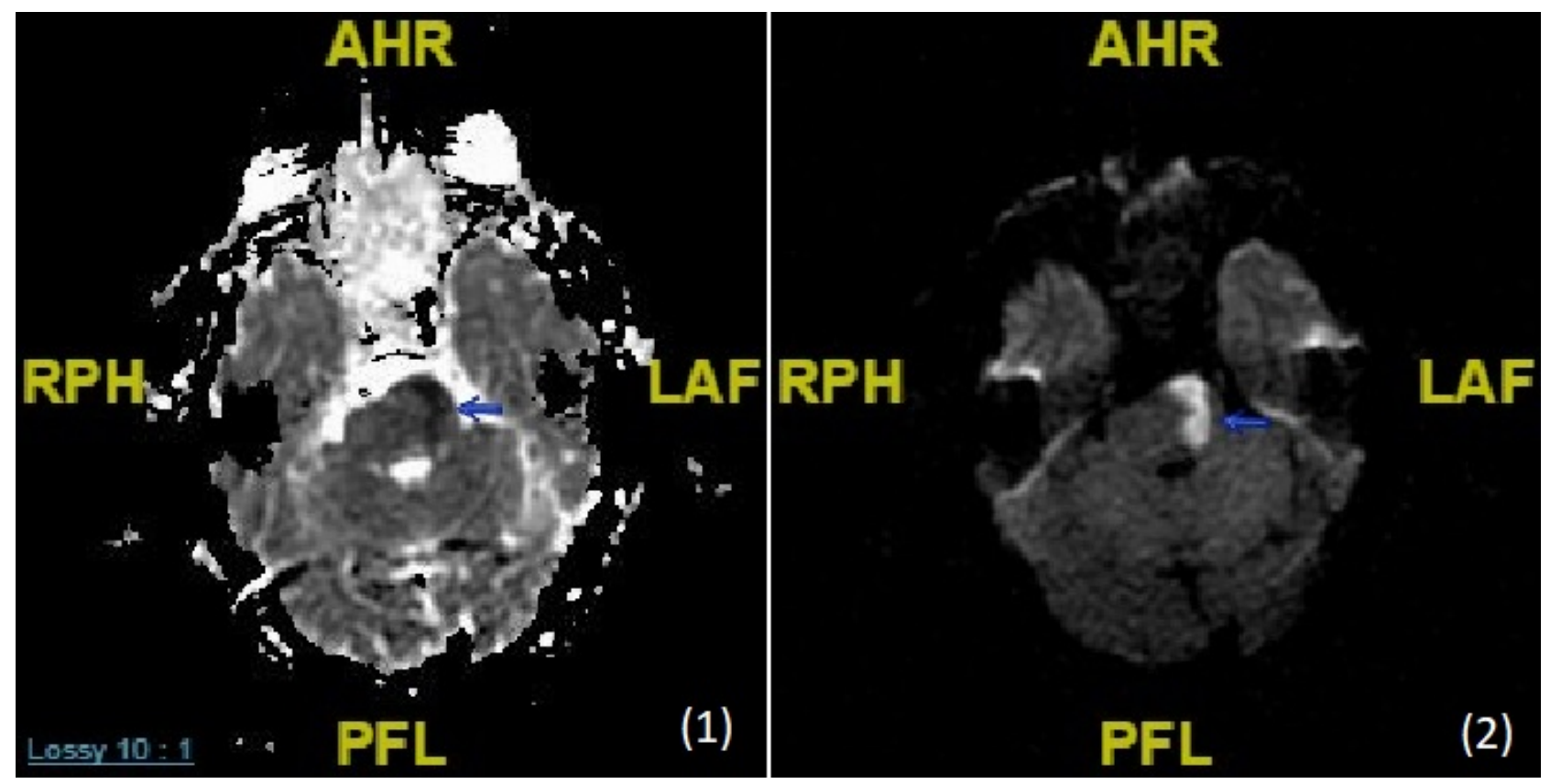

Figure 1: Image compatible to findings of acute ischemia an anterior portion of left hemi-pons on diffusion-weighted MR sequences with corresponding ADC images.

The patient was considered as association of ischemic stroke and CCHF secondary to tick bite. The treatment with ribavirin was promptly started with supportive therapy including platelet transfusion. No antiaggregant therapy for ischemic stroke was given at initial period due to reduction in platelet count. The fever and blood pressure were controlled and supportive therapy was maintained. During follow-up, platelet and leukocyte count was tended to be normalized. In work-up for stroke etiology, no stenosis was detected carotid and vertebral arteries on sonography. In cardiovascular examination, there was no abnormal echocardiography finding other than left ventricular hypertrophy. Since the patient had high blood glucose level, endocrinology consultation was ordered and metformin was initiated. Ataxia was improved and the patient was able to walk without support. Bleeding profile was normal with no novel hemorrhage during follow-up. The patient was discharged by prescribing an antiaggregant agent on the day 10 after admission (Table 1).

\begin{tabular}{|l|l|l|}
\hline & $\begin{array}{l}\text { Laboratory values at } \\
\text { ED presentation } \\
\text { day) }\end{array}$ & $\begin{array}{l}\text { Laboratory values at } \\
\text { discharge (12 day) }\end{array}$ \\
\hline Leukocyte $\left(10^{\wedge} 3 / \mathrm{ML}\right)$ & 970 & 4.15 \\
\hline Platelet $\left(10^{\wedge} 3 / \mathrm{\mu L}\right)$ & 17 & 156 \\
\hline Aptt/sn & 62.2 & 26.9 \\
\hline AST (U/L) & 778 & 101 \\
\hline ALT (U/L) & 425 & 179 \\
\hline LDH (U/L) & 1074 & 260 \\
\hline
\end{tabular}

\begin{tabular}{|l|l|l|}
\hline GGT (U/L) & 410 & 283 \\
\hline CK (U/L) & 883 & 42 \\
\hline Glukoz (mg/dl) & 283 & 176 \\
\hline
\end{tabular}

Table 1: Laboratory values at ED presentation and discharge.

\section{Discussion}

The CCHF is a viral hemorrhagic disease with severe course and high mortality rate, which is caused by Nairovirus belonging to Bunyaviridae family. Domestic animals such as cattle, sheep or goat, and wild animals are reservoir while ticks are both reservoir and vector for CCHF [1].

In Turkey, the CCHF was first detected in Tokat province in 2002. In the same region, 75 cases were detected in 2003. Between 2000 and 2003, CCHF outbreaks were reported in Tokat, Sivas and Yozgat provinces of Middle Anatolian region. Of cases with CCHF, $64.4 \%$ was living in rural areas and female: male ratio was 1:13. There was history of tick exposure in $68.9 \%$ of cases and majority of cases were detected at May, June and July [6]. Our case also presented at May, arising suspicion for CCHF. Thus, CCHF should be questioned in patients presenting with fever between March and October. Our patient was living in Sivas province and working in livestock production.

In previous studies, it was shown that there is increased incidence of stroke in patients with infection older than 50 yrs of age. However, underlying mechanism hasn't been fully elucidated. In addition, it was also shown that metabolic demand is increased in neurons and bloodbrain barrier was disrupted with acidosis and higher amounts of 
excitatory amino acids in patients infected by CCHF. Studies showed that IL-10 release results in delayed and attenuated immune response, which, in turn, causes uncontrolled release of TNF- $\alpha$, IFN- $\gamma$ and cytokines, resulting in promotion of intrinsic pathway of intravascular coagulation $[7,8]$.

Clinical course of CCHF includes incubation, pre-hemorrhagic period, hemorrhagic period and recovery. Incubation period is generally 1-3 days (up to 9 days) after tick bite while it is 5-6 days (up to 13 days) after direct contact withinfected blood, body fluid or tissue. In our patients, symptoms appeared 3 days after contact with infected animal. Flu-like symptoms such as fatigue, myalgia, dizziness, headache, sore throat, photophobia, nausea, vomiting can be seen after incubation period. This may be accompanied by diarrhea and diffuse abdominal pain. There may be confusion, agitation, somnolence and depression in the patients. The disease progresses to hemorrhagic period characterized by bleeding at skin and mucosal tissue. Additional hemorrhagic events such as melena, hematuria, epistaxis or gingival bleeding may be seen in this period. The disease may be fatal in such cases. In survivors, recovery period generally begins 10 to 20 days after onset of disease $[3,9]$. In our patients, there were symptoms including fever, headache, nausea, vomiting and abdominal pain which could be seen in the clinical course of CCHF; in addition, he had imbalance and gait disorder which are seen in stroke. In our case, no hemorrhage was observed during follow-up. In the literature, no clinical finding suggestive of acute cerebrovascular event was detected in previous case reports. This is first case with association of CCHF and ischemic stroke in the literature.

In the laboratory evaluations, leucopenia and thrombocytopenia are striking in CCHF. In biochemistry assays, AST, ALT, creatinine kinase and bilirubin levels are elevated initially; followed by GGT and LDH elevation. Prothrombin time, partial thromboplastin time and other coagulation tests are markedly impaired. In the literature, elevated AST, ALD, LDH and CK levels, prolonged aPTT and PT and decreased platelet count are defined as poor prognostic criteria [10]. In our case, there were elevated hepatic function tests, decreased platelet count, prolonged aPTT and low leukocyte count, meeting poor prognostic criteria. However, no hemorrhage was developed during follow-up and marked improvement was observed in stroke findings. Decreased platelet count and endothelial dysfunction are major causes of bleeding; however, no report was identified suggesting that CCHF leads ischemic stroke in the literature [5]. In our patient, addition of symptoms including imbalance and gait disorder to fever, nausea, vomiting and abdominal pain suggested that inflammatory cascade developed following tick bite resulted in stroke.

Currently, CCHF management includes ribavirin and supportive therapy with fluids, electrolytes, blood and blood products based on severity of disease. Duration of treatment and length of hospital stay are 9-10 days in general. There are many observational studies indicating effectiveness of early ribavirin use in the management of CCHF. Thus, ribavirin was given at early course of disease in our patient [11]. Rapid improvement was observed after ribavirin treatment in our patient. No anticoagulant therapy was given due to low platelet and leukocyte count meeting poor prognostic criteria. Focus was directed to control fever immediately since studies on patients with ischemic stroke showed that body temperature $>39^{\circ} \mathrm{C}$ is associated to increased infarct volume as well as morbidity and mortality [12].

The CCHF should be considered in the differential diagnosis of patients presented with fever, hemorrhagic findings, elevated hepatic enzymes, thrombocytopenia and leukopenia during summer in regions where $\mathrm{CCHF}$ is endemic. Patients with established risk factors such as diabetes, CCHF facilitates the risk of stroke, and these patients are now required to effectively control their primary disease with appropriate treatment. The presenting complaints may be non-specific and there may be no hemorrhagic finding at presentation. We think that a careful neurological examination should be performed at presentation since inflammatory cytokines released during CCHF may lead ischemia as it was the case in our patient.

\section{References}

1. Ergonul O (2008) Crimean-Congo hemorrhagic fever (CCHF) in Turkey: a zoonosis which can cause nosocomial infection: invited commentary. Turkiye Klinikleri Tip Bilimleri Dergisi 28: 677-679.

2. Kara A (2008) Kırım Kongo kanamalı ateşi. Turk Pediatr Arş 43: 108-118.

3. Gunes T, Engin A, Poyraz O, Elaldi N, Kaya S, et al. (2009) CrimeanCongo Hemorrhagic fever virus in high- risk population, Turkey. Emerg Infect Dis 15: 461-464.

4. Yilmaz G, Yilmaz H, Arslan M, Kostakoglu U, Mentese A, et al. (2017) The Prognostic Significance of Serum TGF-b1 Levels in Patients With Crimean-Congo Hemorrhagic Fever. J Med Virol 89: 413-416.

5. Peters CJ, Zaki SR (2002) Role of the endothelium in viral hemorrhagic fevers. Crit Care Med 30: 268-273.

6. Elaldi N, Bodur H, Ascioglu S, Celikbas A, Ozkurt Z, et al. (2009) Efficacy of oral ribavirin treatment in Crimean-Congo haemorrhagic fever: aquasi-experimental study from Turkey. Journal of Infection 58: 238-244.

7. Metanat M, Sharifi-Mood B, Alavi-Naini R, Kermansaravi F, Hamzehnezhad M (2014) Crimean-Congo Hemorrhagic Fever and Hypertention: A Case Report. Arch Clin Infect Dis 9: e20696.

8. Meisel C, Prass K, Braun J, Victorov I, Wolf T, et al. (2004) Preventive antibacterial treatment improves the general medical and neurological outcome in a Mouse model of stroke. Stroke 35: 2-6.

9. Ergonul O (2006) Crimean-Congo haemorrhagic fever. Lancet Infect Dis 6: 203-214.

10. Yadav PD, Thacker S, Patil DY, Jain R, Mourya DT (2017) CrimeanCongo Hemorrhagic Fever in Migrant Worker Returning from Oman to India, 2016. Emerg Infect Dis 23: 1005-1008.

11. Gowen BB, Hickerson BT (2017) Hemorrhagic fever of bunyavirus etiology: disease models and progress towards new therapies. J Microbiol 55: 183-195.

12. Boysen G, Christensen H (2001) Stroke severity determines body temperature in acute stroke. Stroke 32: 413- 417. 